\title{
Article
}

\section{An Optimal Derivative Free Family of Chebyshev-Halley's Method for Multiple Zeros}

\author{
Ramandeep Behl ${ }^{1,+}$, Sonia Bhalla ${ }^{2,+}$, Ángel Alberto Magreñán ${ }^{3, *,+}+\mathbb{D}$ and Alejandro Moysi ${ }^{3,+}$ \\ 1 Department of Mathematics, King Abdulaziz University, Jeddah 21589, Saudi Arabia; ramanbehl87@yahoo.in \\ 2 Department of Mathematics, Chandigarh University, Gharuan, Mohali 140413, Punjab, India; \\ sonia.e8843@cumail.in \\ 3 Department of Mathematics and Mathematics, University of La Rioja, Madre de Dios 53, \\ 26006 Logroño (La Rioja), Spain; alejandro.moysi@alum.unirioja.es \\ * Correspondence: angel-alberto.magrenan@unirioja.es \\ + All authors contributed equally to this work.
}

check for updates

Citation: Behl, R.; Bhalla, S.;

Magreñán, Á.A.; Moysi, A. An Optimal Derivative Free Family of Chebyshev-Halley's Method for Multiple Zeros. Mathematics 2021, 9 , 546. https://doi.org/10.3390/ math9050546

Academic Editor: Ravi P. Agarwal

Received: 20 January 2021

Accepted: 3 March 2021

Published: 5 March 2021

Publisher's Note: MDPI stays neutral with regard to jurisdictional claims in published maps and institutional affiliations.

\begin{abstract}
In this manuscript, we introduce the higher-order optimal derivative-free family of Chebyshev-Halley's iterative technique to solve the nonlinear equation having the multiple roots. The designed scheme makes use of the weight function and one parameter $\alpha$ to achieve the fourthorder of convergence. Initially, the convergence analysis is performed for particular values of multiple roots. Afterward, it concludes in general. Moreover, the effectiveness of the presented methods are certified on some applications of nonlinear equations and compared with the earlier derivative and derivative-free schemes. The obtained results depict better performance than the existing methods.
\end{abstract}

Keywords: nonlinear equations; Kung-Traub conjecture; multiple roots; optimal iterative methods; efficiency index

\section{Introduction}

Most scientific and engineering problems involve nonlinear equations. For the evaluation of the roots of such nonlinear equations, analytical methods are almost nonexistent. This leads to the inspiration for the solution of nonlinear equations with iterative techniques. The well-known iterative techniques for solving nonlinear equations are Newton's method and Chebyshev-Halley. The order of convergence of Newton's method is quadratic whereas Chebyshev-Halley methods have cubic order of convergence. Later on, many higher-order variants of Newton's method and Chebyshev-Halley family were developed to solve nonlinear equations. In literature, plenty of iterative techniques [1-9] involving first-order derivative are available to find the roots of nonlinear equation $g(x)=0$ with multiplicity $m \geq 2$ that is satisfying the conditions $g^{\prime}(x)=g^{\prime \prime}(x)=$ $\cdots=g^{m-1}(x)=0$ and $g^{m}(x) \neq 0$. One of the established technique is modified Newton's method [10-12] which is defined as

$$
x_{\lambda+1}=x_{\lambda}-m \frac{g\left(x_{\lambda}\right)}{g^{\prime}\left(x_{\lambda}\right)} .
$$

The order of convergence of modified Newton's method is quadratic for $m \geq 1$ and requires the computation of first-order derivative. Some of the other techniques like Schröder iterative method [11], Chebyshev-Halley method [13] consists of the secondorder derivative of a function which increases the computation cost. On the other hand, a few literature is investigated to the construction of derivative free methods to find the multiple zeros of nonlinear equations. To reduce the derivative computation of a function, authors have used the well known Traub-Steffensen method [11] for multiple roots which is defined as:

$$
x_{\lambda+1}=x_{\lambda}-m \frac{g\left(x_{\lambda}\right)}{g\left[\mu_{\lambda}, x_{\lambda}\right]},
$$


where $\mu_{\lambda}=x_{\lambda}+\gamma g\left(x_{\lambda}\right), \quad \gamma \in \mathbb{R}$ and $g\left[\mu_{\lambda}, x_{\lambda}\right]=\frac{g\left(\mu_{\lambda}\right)-g\left(x_{\lambda}\right)}{\mu_{\lambda}-x_{\lambda}}$.

In 2015, Hueso et al. [14] introduced the fourth-order derivative free technique to solve nonlinear equations with multiple roots. In this scheme first order derivative is approximated with divided difference operator shown as:

$$
g^{\prime}(x) \approx g\left[\mu_{\lambda}, x_{\lambda}\right]
$$

where $\mu_{\lambda}=x_{\lambda}+g\left(x_{\lambda}\right)^{q}, q \in \mathbb{R}$.

Presently, Kumar et al. [15] and Sharma et al. [16-18] developed derivative-free methods of second-order, fourth-order, and eighth-order convergence respectively. Moreover, these methods are optimal as it satisfies the Kung-Traub conjecture [19] means the order of convergence is $2^{n-1}$ where $n$ represents the number of function evaluations per iteration. Inspired from this, we have constructed an optimal derivative-free Chebyshev-Halley family for multiple roots of a nonlinear equation. Our method consists of three functional evaluations, one weighted function $H(\tau)$, and one parameter $\alpha$ to accomplish better computational efficiency and fourth-order convergence. Further, with different choices of the weighted function $H(\tau)$, and parameter $\alpha$ many methods can be generated.

The summary of the sections is as follows. In Section 2, the construction of the higher-order method is elaborated. The convergence analysis of the designed method is presented in Section 2. Some special cases of the proposed method are highlighted in Section 3. In Section 4, numerical experiments are performed. Finally, Section 5 concludes the presented work.

\section{Construction of Higher-Order Scheme}

Here, we construct an optimal fourth-order family of iterative method for multiple zeros $m \geq 2$ with simple and compact body structure, which is defined by

$$
\begin{aligned}
y_{\lambda} & =x_{\lambda}-m \frac{g\left(x_{\lambda}\right)}{g\left[\mu_{\lambda}, x_{\lambda}\right]}, \\
x_{\lambda+1} & =x_{\lambda}+m \frac{g\left(x_{\lambda}\right)}{g\left[\mu_{\lambda}, x_{\lambda}\right]}\left(1+\frac{\zeta}{1-2 \alpha \zeta}\right)\left[\frac{1}{2} \zeta-H(\tau)\right], \quad \alpha \in \mathbb{R},
\end{aligned}
$$

where $\mu_{\lambda}=x_{\lambda}+\gamma g\left(x_{\lambda}\right), \quad \gamma \in \mathbb{R}$ is any finite real number and $m \geq 2$ is the known multiplicity of the required zero. In addition, the function $H: \mathbb{C} \rightarrow \mathbb{C}$ is analytic in the neighborhood of origin (0). Moroever, we considered $\tau=\left(\frac{g\left(y_{\lambda}\right)}{g\left(\mu_{\lambda}\right)}\right)^{\frac{1}{m}}$ and $\zeta=\left(\frac{g\left(y_{\lambda}\right)}{g\left(x_{\lambda}\right)}\right)^{\frac{1}{m}}$ two multi-valued functions. Suppose their principal analytic branches (see $[7,20]), v$ as a principal root given by $\tau=\exp \left[\frac{1}{m} \log \left(\frac{g\left(y_{\lambda}\right)}{g\left(\mu_{\lambda}\right)}\right)\right]$, with $\log \left(\frac{g\left(y_{\lambda}\right)}{g\left(\mu_{\lambda}\right)}\right)=\log \left|\frac{g\left(y_{\lambda}\right)}{g\left(\mu_{\lambda}\right)}\right|+$ $i \arg \left(\frac{g\left(y_{\lambda}\right)}{g\left(\mu_{\lambda}\right)}\right)$ for $-\pi<\arg \left(\frac{g\left(y_{\lambda}\right)}{g\left(\mu_{\lambda}\right)}\right) \leq \pi$. The choice of $\arg (z)$ for $z \in \mathbb{C}$ agrees with that of $\log (z)$ to be employed later in numerical experiments of Section 4 . We have an analogous way $\zeta=\left|\frac{g\left(y_{\lambda}\right)}{g\left(x_{\lambda}\right)}\right|^{\frac{1}{m}} \cdot \exp \left[\frac{1}{m} \arg \left(\frac{g\left(y_{\lambda}\right)}{g\left(x_{\lambda}\right)}\right)\right]=O\left(e_{\lambda}\right)$.

In Theorem 1, we illustrate that the constructed Scheme (4) attains maximum fourthorder of convergence for multiplicity $m=2$ zeros and for all $\alpha, \gamma \in \mathbb{R}$, without adopting any supplementary evaluation of function or its derivative.

Theorem 1. Suppose $\eta$ is a solution of multiplicity $m=2$ of function $g$. Consider that function $g: \mathbb{D} \subset \mathbb{C} \rightarrow \mathbb{C}$ is an analytic in $\mathbb{D}$ surrounding the required zero $\eta$. Then, the presented Scheme (4) has fourth-order convergence, provided

$$
H(0)=1, \quad H^{\prime}(0)=\frac{1}{2}, \quad H^{\prime \prime}(0)=4(1-\alpha)
$$


and satisfies the following error equation

$$
\begin{aligned}
e_{\lambda+1}= & -\frac{\left(\gamma g^{\prime \prime}(\eta)+2 a_{1}\right)}{384}\left[\gamma^{2}\left(g^{\prime \prime}(\eta)\right)^{2}\left(24 \alpha^{2}+36 \alpha+\theta-42\right)\right. \\
& +4 \gamma g^{\prime \prime}(\eta)\left(12\left(2 \alpha^{2}+g^{\prime \prime}(\eta)-2\right)+\theta\right) a_{1} \\
& \left.+4\left(3\left(8 \alpha^{2}-4 \alpha-7\right)+\theta\right) a_{1}^{2}+48 a_{2}\right] e_{\lambda}^{4}+O\left(e_{\lambda}^{5}\right),
\end{aligned}
$$

where $\theta=H^{\prime \prime \prime}(0) \in \mathbb{R}$.

Proof. Let $e_{\lambda}=x_{\lambda}-\eta$ and $a_{k}=\frac{2 !}{(2+k) !} \frac{g^{(2+k)}(\eta)}{g^{(2)}(\eta)}, k=1,2,3,4$ be the error in $\lambda$-th iteration and asymptotic error constant numbers, respectively. We use the Taylor's series expansions for $g\left(x_{\lambda}\right)$ and $g\left(\mu_{\lambda}\right)$ around $x=\eta$ with the assumption $g(\eta)=g^{\prime}(\eta)=0$ and $g^{\prime \prime}(\eta) \neq 0$, which are given by

$$
g\left(x_{\lambda}\right)=\frac{g^{\prime \prime}(\eta)}{2 !} e_{\lambda}^{2}\left(1+a_{1} e_{\lambda}+a_{2} e_{\lambda}^{2}+a_{3} e_{\lambda}^{3}+a_{4} e_{\lambda}^{4}+O\left(e_{\lambda}^{5}\right)\right)
$$

and

$$
\begin{aligned}
g\left(\mu_{\lambda}\right)= & \frac{g^{\prime \prime}(\eta)}{2 !} e_{\lambda}^{2}\left[1+\left(\gamma g^{\prime \prime}(\eta)+a_{1}\right) e_{\lambda}+\frac{1}{4}\left\{\gamma^{2}\left(g^{\prime \prime}(\eta)\right)^{2}+10 \gamma g^{\prime \prime}(\eta) a_{1}+4 a_{2}\right\} e_{\lambda}^{2}\right. \\
& +\frac{1}{4}\left\{5 \gamma^{2}\left(g^{\prime \prime}(\eta)\right)^{2} a_{1}+6 \gamma g^{\prime \prime}(\eta) a_{1}^{2}+12 \gamma g^{\prime \prime}(\eta) a_{2}+4 a_{3}\right\} e_{\lambda}^{3} \\
& +\frac{1}{8}\left\{\gamma^{3}\left(g^{\prime \prime}(\eta)\right)^{3} a_{1}+14 \gamma^{2}\left(g^{\prime \prime}(\eta)\right)^{2} a_{1}^{2}+16 \gamma^{2}\left(g^{\prime \prime}(\eta)\right)^{2} a_{2}+28 \gamma g^{\prime \prime}(\eta) a_{1} a_{2}\right. \\
& \left.\left.+28 \gamma g^{\prime \prime}(\eta) a_{3}+8 a_{4}\right\} e_{\lambda}^{4}+O\left(e_{\lambda}^{5}\right)\right],
\end{aligned}
$$

respectively.

We obtain the following expression by adopting (6) and (7) in the Scheme (4)

$$
\begin{aligned}
y_{\lambda}-\eta & =\frac{1}{4}\left(\gamma g^{\prime \prime}(\eta)+2 a_{1}\right) e_{\lambda}^{2}-\frac{1}{16}\left[\gamma^{2}\left(g^{\prime \prime}(\eta)\right)^{2}-8 \gamma g^{\prime \prime}(\eta) a_{1}+12 a_{1}^{2}-16 a_{2}\right] e_{\lambda}^{3} \\
& +\frac{1}{64}\left[\gamma^{3}\left(g^{\prime \prime}(\eta)\right)^{3}-10 a_{1}\left(\gamma^{2}\left(g^{\prime \prime}(\eta)\right)^{2}+16 a_{2}\right)-20 \gamma g^{\prime \prime}(\eta) a_{1}^{2}+64 \gamma g^{\prime \prime}(\eta) a_{2}\right. \\
& \left.+72 a_{1}^{3}+96 a_{3}\right] e_{\lambda}^{4}+O\left(e_{\lambda}^{5}\right) .
\end{aligned}
$$

We have the following $g\left(y_{\lambda}\right)$ by using Taylor series expansion and expression (8)

$$
\begin{aligned}
g\left(y_{\lambda}\right)= & \frac{g^{\prime \prime}(\eta)}{2 !} e_{\lambda}^{2}\left[\frac{1}{16}\left(\gamma g^{\prime \prime}(\eta)+2 a_{1}\right)^{2} e_{\lambda}^{2}-\frac{1}{32}\left(\gamma g^{\prime \prime}(\eta)+2 a_{1}\right)\right. \\
& \times\left(\gamma^{2}\left(g^{\prime \prime}(\eta)\right)^{2}-8 \gamma g^{\prime \prime}(\eta) a_{1}+12 a_{1}^{2}-16 a_{2}\right) e_{\lambda}^{3}+\frac{1}{256}\left\{3 \gamma^{4}\left(g^{\prime \prime}(\eta)\right)^{4}\right. \\
& -4 a_{1}\left(7 \gamma^{3}\left(g^{\prime \prime}(\eta)\right)^{3}-48 \gamma g^{\prime \prime}(\eta) a_{2}-96 a_{3}\right)+96 \gamma^{2}\left(g^{\prime \prime}(\eta)\right)^{2} a_{2}-80 \gamma g^{\prime \prime}(\eta) a_{1}^{3} \\
& \left.\left.+32 a_{1}^{2}\left(\gamma^{2}\left(g^{\prime \prime}(\eta)\right)^{2}-32 a_{2}\right)+192 \gamma g^{\prime \prime}(\eta) a_{3}+464 a_{1}^{4}+256 a_{2}^{2}\right\} e_{\lambda}^{4}+O\left(e_{\lambda}^{5}\right)\right] .
\end{aligned}
$$

From the Equations (6)-(9), we further deduce

$$
\zeta=\left(\frac{g\left(y_{\lambda}\right)}{g\left(x_{\lambda}\right)}\right)^{\frac{1}{2}}=\frac{1}{4}\left(\gamma g^{\prime \prime}(\eta)+2 a_{1}\right) e_{\lambda}+v_{1} e_{\lambda}^{2}+v_{2} e_{\lambda}^{3}+O\left(e_{\lambda}^{4}\right)
$$

and

$$
\tau=\left(\frac{g\left(y_{\lambda}\right)}{g\left(\mu_{\lambda}\right)}\right)^{\frac{1}{2}}=\frac{1}{4}\left(\gamma g^{\prime \prime}(\eta)+2 a_{1}\right) e_{\lambda}+v_{3} e_{\lambda}^{2}+v_{4} e_{\lambda}^{3}+O\left(e_{\lambda}^{4}\right)
$$


where

$$
\begin{aligned}
& v_{1}=\frac{-\gamma^{3}\left(g^{\prime \prime}(\eta)\right)^{3}+4 \gamma^{2}\left(g^{\prime \prime}(\eta)\right)^{2} a_{1}-4 \gamma g^{\prime \prime}(\eta) a_{1}^{2}+16 \gamma g^{\prime \prime}(\eta) a_{2}-32 a_{1}^{3}+32 a_{1} a_{2}}{16\left(\gamma g^{\prime \prime}(\eta)+2 a_{1}\right)}, \\
& v_{2}=\frac{1}{64}\left(\gamma^{3}\left(g^{\prime \prime}(\eta)\right)^{3}-6 \gamma^{2}\left(g^{\prime \prime}(\eta)\right)^{2} a_{1}-22 \gamma g^{\prime \prime}(\eta) a_{1}^{2}+56 \gamma g^{\prime \prime}(\eta) a_{2}+116 a_{1}^{3}-208 a_{1} a_{2}+96 a_{3}\right), \\
& v_{3}=\frac{-3 \gamma^{3}\left(g^{\prime \prime}(\eta)\right)^{3}-4 \gamma^{2}\left(g^{\prime \prime}(\eta)\right)^{2} a_{1}-12 \gamma g^{\prime \prime}(\eta) a_{1}^{2}+16 \gamma g^{\prime \prime}(\eta) a_{2}-32 a_{1}^{3}+32 a_{1} a_{2}}{16\left(\gamma g^{\prime \prime}(\eta)+2 a_{1}\right)}, \\
& v_{4}=\frac{1}{64}\left(7 \gamma^{3}\left(g^{\prime \prime}(\eta)\right)^{3}-22 \gamma^{2}\left(g^{\prime \prime}(\eta)\right)^{2} a_{1}-14 \gamma g^{\prime \prime}(\eta) a_{1}^{2}+24 \gamma g^{\prime \prime}(\eta) a_{2}+116 a_{1}^{3}-208 a_{1} a_{2}+96 a_{3}\right) .
\end{aligned}
$$

The expression (11) demonstrates that the $\tau$ is of order one $\left(\tau=O\left(e_{\lambda}\right)\right)$. Then, we can expand the weight function $H(\tau)$ in the neighborhood of origin in the following way:

$$
H(\tau)=H(0)+H^{\prime}(0) \tau+\frac{1}{2 !} H^{\prime \prime}(0) \tau^{2}+\frac{1}{3 !} H^{\prime \prime \prime}(0) \tau^{3} .
$$

We have the following expression by inserting Equations (6)-(12) in the Scheme (4)

$$
e_{\lambda+1}=(1-H(0)) e_{\lambda}+\sum_{i=1}^{3} \Omega_{i} e_{\lambda}^{i+1}+O\left(e_{\lambda}^{5}\right)
$$

where $\Omega_{i}=\Omega_{i}\left(g^{\prime \prime}(\eta), \alpha, \gamma, a_{1}, a_{2}, a_{3}, a_{4}, H(0), H^{\prime}(0), H^{\prime \prime}(0), H^{\prime \prime \prime}(0)\right)$. For Example, $\Omega_{1}=$ $-\frac{1}{8}\left(2 H^{\prime}(0)-1\right)\left(\gamma g^{\prime \prime}(\eta)+2 a_{1}\right)$, etc.

From the expression (13), we deduce that the Scheme (4) reaches at least second-order convergence, if

$$
H(0)=0 .
$$

Adopting the expression (14) and $\Omega_{1}=0$, we obtain

$$
-\frac{1}{8}\left(2 H^{\prime}(0)-1\right)\left(\gamma g^{\prime \prime}(\eta)+2 a_{1}\right)=0,
$$

which further gives

$$
H^{\prime}(0)=\frac{1}{2}
$$

By inserting expressions (14) and (16) in $\Omega_{2}=0$, we have

$$
-\frac{1}{32}\left(4 \alpha+H^{\prime \prime}(0)-4\right)\left(\gamma g^{\prime \prime}(\eta)+2 a_{1}\right)^{2}=0,
$$

which further leads us to

$$
H^{\prime \prime}(0)=4(1-\alpha) \text {. }
$$

Next, by inserting (14), (16) and (18) in (13), we get

$$
\begin{aligned}
e_{\lambda+1}= & -\frac{\left(\gamma g^{\prime \prime}(\eta)+2 a_{1}\right)}{384}\left[\gamma^{2}\left(g^{\prime \prime}(\eta)\right)^{2}\left(24 \alpha^{2}+36 \alpha+\theta-42\right)\right. \\
& +4 \gamma g^{\prime \prime}(\eta)\left(12\left(2 \alpha^{2}+g^{\prime \prime}(\eta)-2\right)+\theta\right) a_{1} \\
& \left.+4\left(3\left(8 \alpha^{2}-4 \alpha-7\right)+\theta\right) a_{1}^{2}+48 a_{2}\right] e_{\lambda}^{4}+O\left(e_{\lambda}^{5}\right),
\end{aligned}
$$

where $\theta=H^{\prime \prime \prime}(0) \in \mathbb{R}$. Hence, the Scheme (4) has fourth-order convergence for $m=2$.

Theorem 2. Adopting the same hypotheses of Theorem 1, the scheme given by (4) is of fourth-order convergence for $m=3$. It satisfies the following error equation

$$
e_{\lambda+1}=-\frac{b_{1}}{162}\left[\left(24 \alpha^{2}-12 \alpha+\theta-24\right) b_{1}^{2}+\frac{9}{2} \gamma g^{\prime \prime \prime}(\eta)+18 b_{2}\right] e_{\lambda}^{4}+O\left(e_{\lambda}^{5}\right),
$$


where $b_{j}=\frac{3 !}{(3+j) !} \frac{g^{(3+j)}(\eta)}{g^{(3)}(\eta)}, j=1,2,3,4$.

Proof. We use the Taylor's series expansions for $g\left(x_{\lambda}\right)$ and $g\left(\mu_{\lambda}\right)$ around $x=\eta$ with the assumption $g(\eta)=g^{\prime}(\eta)=g^{\prime \prime}(\eta)=0$ and $g^{\prime \prime \prime}(\eta) \neq 0$, which are defined as follow:

$$
g\left(x_{\lambda}\right)=\frac{g^{\prime \prime \prime}(\eta)}{3 !} e_{\lambda}^{3}\left(1+b_{1} e_{\lambda}+b_{2} e_{\lambda}^{2}+b_{3} e_{\lambda}^{3}+b_{4} e_{\lambda}^{4}+O\left(e_{\lambda}^{5}\right)\right)
$$

and

$$
\begin{aligned}
g\left(\mu_{\lambda}\right)= & \frac{g^{\prime \prime \prime}(\eta)}{3 !} e_{\lambda}^{3}\left[1+b_{1} e_{\lambda}+\frac{1}{2}\left(\gamma g^{\prime \prime \prime}(\eta)+2 b_{2}\right) e_{\lambda}^{2}+\left(\frac{7}{6} \gamma g^{\prime \prime \prime}(\eta) b_{1}+b_{3}\right) e_{\lambda}^{3}\right. \\
& \left.+\frac{1}{72}\left(\gamma^{2}\left(g^{\prime \prime \prime}(\eta)\right)^{2}+8 \gamma g^{\prime \prime \prime}(\eta) T_{1}^{2}+16 \gamma g^{\prime \prime \prime}(\eta) T_{2}+12 T_{4}\right) e_{\lambda}^{4}+O\left(e_{\lambda}^{5}\right)\right] .
\end{aligned}
$$

Adopting expressions (20) and (21) in the Scheme (4), we get

$$
\begin{aligned}
y_{\lambda}-\eta & =\frac{b_{1}}{3} e_{\lambda}^{2}+\frac{1}{18}\left(3 \gamma g^{\prime \prime \prime}(\eta)-8 b_{1}^{2}+12 b_{2}\right) e_{\lambda}^{3}+\left\{\frac{1}{9} b_{1}\left(2 \gamma g^{\prime \prime \prime}(\eta)-13 b_{2}\right)\right. \\
& \left.+\frac{16 b_{1}^{3}}{27}+b_{3}\right\} e_{\lambda}^{4}+O\left(e_{\lambda}^{5}\right) .
\end{aligned}
$$

We have the following $g\left(y_{\lambda}\right)$ by using the Taylor series expansion and expression (22)

$$
g\left(y_{\lambda}\right)=\frac{g^{\prime \prime \prime}(\eta)}{3 !} e_{\lambda}^{3}\left[\frac{b_{1}^{3}}{27} e_{\lambda}^{3}+\frac{1}{54} b_{1}^{2}\left(3 \gamma g^{\prime \prime \prime}(\eta)-8 b_{1}^{2}+12 b_{2}\right) e_{\lambda}^{4}+O\left(e_{\lambda}^{5}\right)\right] .
$$

The expressions (20), (21) and (23), leads us to

$$
\begin{aligned}
\zeta=\left(\frac{g\left(y_{\lambda}\right)}{g\left(x_{\lambda}\right)}\right)^{\frac{1}{3}} & =\frac{b_{1}}{3} e_{\lambda}+\frac{1}{18 b_{1}^{2}}\left(3 \gamma g^{\prime \prime \prime}(\eta) b_{1}^{2}-10 b_{1}^{4}+12 b_{2} b_{1}^{2}\right) e_{\lambda}^{2}+\frac{1}{54}\left(9 \gamma g^{\prime \prime \prime}(\eta) b_{1}\right. \\
& \left.+46 b_{1}^{3}-96 b_{2} b_{1}+54 b_{3}\right) e_{\lambda}^{3}+O\left(e_{\lambda}^{4}\right)
\end{aligned}
$$

and

$$
\begin{aligned}
\tau=\left(\frac{g\left(y_{\lambda}\right)}{g\left(\mu_{\lambda}\right)}\right)^{\frac{1}{3}}= & \frac{b_{1}}{3} e_{\lambda}+\frac{1}{18 b_{1}^{2}}\left(3 \gamma g^{\prime \prime \prime}(\eta) b_{1}^{2}-10 b_{1}^{4}+12 b_{2} b_{1}^{2}\right) e_{\lambda}^{2} \\
& +\frac{1}{27}\left(3 \gamma g^{\prime \prime \prime}(\eta) b_{1}+23 b_{1}^{3}-48 b_{2} b_{1}\right. \\
& \left.+27 b_{3}\right) e_{\lambda}^{3}+O\left(e_{\lambda}^{4}\right) .
\end{aligned}
$$

Clearly, the expression (25) is of order one $\left(\tau=O\left(e_{\lambda}\right)\right)$. Then, we have

$$
H(\tau)=H(0)+H^{\prime}(0) \tau+\frac{1}{2 !} H^{\prime \prime}(0) \tau^{2}+\frac{1}{3 !} H^{\prime \prime \prime}(0) \tau^{3} .
$$

Adopting (20)-(26) in the Scheme (4), we obtain

$$
e_{\lambda+1}=(1-H(0)) e_{\lambda}+\sum_{i=1}^{3} \Gamma_{i} e_{\lambda}^{i+1}+O\left(e_{\lambda}^{5}\right)
$$

where $\Gamma_{i}=\Gamma_{i}\left(g^{\prime \prime \prime}(\eta), \alpha, \gamma, b_{1}, b_{2}, b_{3}, b_{4}, H(0), H^{\prime}(0), H^{\prime \prime}(0), H^{\prime \prime \prime}(0)\right)$ i.e., $\Gamma_{1}=\frac{1}{6}\left(1-2 H^{\prime}(0)\right)$ $b_{1}$, etc. 
From the expression (27), we deduce that the Scheme (4) attains minimum secondorder convergence, if

$$
H(0)=1 \text {. }
$$

By using expression (28) and $\Gamma_{1}=0$, we get

$$
\frac{1}{6}\left(1-2 H^{\prime}(0)\right) b_{1}=0,
$$

which further leads us to

$$
H^{\prime}(0)=\frac{1}{2}
$$

Adopting the expressions (28) and (30) in $\Gamma_{2}=0$, we obtain

$$
-\frac{1}{18}\left(4 \alpha+H^{\prime \prime}(0)-4\right) b_{1}^{2}=0
$$

that further yields

$$
H^{\prime \prime}(0)=4(1-\alpha) \text {. }
$$

Next, by using (28), (30) and (32) in (27), we yields

$$
e_{\lambda+1}=-\frac{b_{1}}{162}\left[\left(24 \alpha^{2}-12 \alpha+\theta-24\right) b_{1}^{2}+\frac{9}{2} \gamma g^{\prime \prime \prime}(\eta)+18 b_{2}\right] e_{\lambda}^{4}+O\left(e_{\lambda}^{5}\right) .
$$

Hence, the Scheme (4) has fourth-order convergence for $m=3$.

Error for the General Form of the Scheme (4)

Theorem 3. Adopting the same hypotheses of Theorem 1, the scheme given by (4) is of fourth-order convergence for $m \geq 4$. It converges to the following error equation

$$
e_{\lambda+1}=-\frac{c_{1}}{6 m^{3}}\left[\left(24 \alpha^{2}-12 \alpha+\theta-(3 m+15)\right) c_{1}^{2}+6 m c_{2}\right] e_{\lambda}^{4}+O\left(e_{\lambda}^{5}\right) .
$$

Proof. Let $e_{\lambda}=x_{\lambda}-\eta$ and $c_{k}=\frac{m !}{(m+k) !} \frac{g^{(m+k)}(\eta)}{g^{(m)}(\eta)}, k=1,2,3,4$ be the errors in $\lambda$ th iteration and asymptotic error constant numbers, respectively. We use the Taylor's series expansions for the functions $g\left(x_{\lambda}\right)$ and $g\left(\mu_{\lambda}\right)$ around $x=\eta$, which are given by respectively

$$
g\left(x_{\lambda}\right)=\frac{g^{(m)}(\eta)}{m !} e_{\lambda}^{m}\left(1+c_{1} e_{\lambda}+c_{2} e_{\lambda}^{2}+c_{3} e_{\lambda}^{3}+c_{4} e_{\lambda}^{4}+O\left(e_{\lambda}^{5}\right)\right)
$$

and

$$
g\left(\mu_{\lambda}\right)=\frac{g^{m}(\eta)}{m !} e_{\lambda}^{m}\left[1+\sum_{i=1}^{3} \Delta_{i} e_{\lambda}^{i+1}+O\left(e_{\lambda}^{5}\right)\right]
$$

with the assumption $g(\eta)=g^{\prime}(\eta)=g^{(m-1)}(\eta)=0$ and $g^{(m)}(\eta) \neq 0$ and $\Delta_{i}=\Delta_{i}\left(m, g^{(m)}(\eta), \gamma, c_{1}, c_{2}, c_{3}, c_{4}\right)$. For Example, $\Delta_{1}=c_{1}, \quad \Delta_{2}=c_{2}$ and $\Delta_{3}=\left\{\begin{array}{ll}\frac{g^{(4)}(\eta)}{6}\left(\gamma g^{(4)}(\eta)+6 c_{3}\right), & m=4 \\ c_{3}, & m \geq 5\end{array}\right\}$, etc.

We obtain the following expression by adopting (34) and (35) in the Scheme (4)

$$
\begin{aligned}
e_{y_{\lambda}}=y_{\lambda}-\eta & =\frac{c_{1}}{m} e_{\lambda}^{2}+\frac{1}{m^{2}}\left(2 m c_{2}-(1+m) c_{1}^{2}\right) e_{\lambda}^{3}+\frac{1}{m^{3}}\left(3 m^{2} c_{3}\right. \\
& \left.+(m+1)^{2} c_{1}^{3}-m(3 m+4) c_{2} c_{1}\right) e_{\lambda}^{4}+O\left(e_{\lambda}^{5}\right) .
\end{aligned}
$$


We have the following $g\left(y_{\lambda}\right)$ by using expression (36) and Taylor series expansion

$$
g\left(y_{\lambda}\right)=\frac{g^{(m)}(\eta)}{m !} e_{y_{\lambda}}^{m}\left[1+c_{1} e_{y_{\lambda}}+c_{2} e_{y_{\lambda}}^{2}+c_{3} e_{y_{\lambda}}^{3}+c_{4} e_{y_{\lambda}}^{4}+O\left(e_{\lambda}^{5}\right)\right] .
$$

From the expressions (34), (35) and (37), we further deduce

$$
\begin{aligned}
\zeta=\left(\frac{g\left(y_{\lambda}\right)}{g\left(x_{\lambda}\right)}\right)^{\frac{1}{m}}= & \frac{c_{1}}{m} e_{\lambda}+\left(\frac{2}{m} c_{2}-\frac{(m+2)}{m^{2}} c_{1}^{2}\right) e_{\lambda}^{2} \\
& +\frac{1}{2 m^{3}}\left[\left(2 m^{2}+7 m+7\right) c_{1}^{3}-2 m(3 m+7) c_{1} c_{2}+6 m^{2} c_{3}\right] e_{\lambda}^{3} \\
& +\frac{1}{3 m^{4}}\left[-\left(\frac{11 m^{2}}{2}+\frac{33 m}{2}+14\right) c_{1}^{4}-12 m^{2} c_{3} c_{1}-6 m^{2} c_{2}^{2}\right. \\
& \left.+3 m(6 m+11) c_{2} c_{1}^{2}\right] e_{\lambda}^{4}+O\left(e_{\lambda}^{5}\right)
\end{aligned}
$$

and

$$
\begin{aligned}
\tau=\left(\frac{g\left(y_{\lambda}\right)}{g\left(\mu_{\lambda}\right)}\right)^{\frac{1}{m}}= & \frac{c_{1}}{m} e_{\lambda}+\frac{1}{m^{2}}\left(2 m c_{2}-(m+2) c_{1}^{2}\right) e_{\lambda}^{2}+\frac{1}{2 m^{3}}\left(\left(2 m^{2}+7 m+7\right) c_{1}^{3}\right. \\
& \left.-2 m(3 m+7) c_{1} c_{2}+6 m^{2} c_{3}\right) e_{\lambda}^{3}+O\left(e_{\lambda}^{4}\right) .
\end{aligned}
$$

Clearly the expression (39), is of order one $\left(\tau=O\left(e_{\lambda}\right)\right)$, that leads us to

$$
H(\tau)=H(0)+H^{\prime}(0) \tau+\frac{1}{2 !} H^{\prime \prime}(0) \tau^{2}+\frac{1}{3 !} H^{\prime \prime \prime}(0) \tau^{3} .
$$

We have by inserting (34)-(40) in the Scheme (4)

$$
e_{\lambda+1}=(1-H(0)) e_{\lambda}+\sum_{i=1}^{3} \phi_{i} e_{\lambda}^{i+1}+O\left(e_{\lambda}^{5}\right)
$$

where $\phi_{i}=\phi_{i}\left(m, g^{(m)}(\eta), \gamma, \alpha, c_{1}, c_{2}, c_{3}, c_{4}, H(0), H^{\prime}(0), H^{\prime \prime}(0), H^{\prime \prime \prime}(0)\right)$ i.e., $\phi_{1}=\frac{1}{2 m}(1-$ $\left.2 H^{\prime}(0)\right)$, etc.

From the expression (41), we deduce that the Scheme (4) attains at least second-order convergence, if

$$
H(0)=1
$$

Adopting the expression (42) and $\phi_{1}=0$, we get

$$
\frac{1}{2 m}\left(1-2 H^{\prime}(0)\right)=0
$$

that further gives

$$
H^{\prime}(0)=\frac{1}{2}
$$

We obtain the following expression by adopting (42) and (44) in $\phi_{2}=0$

$$
-\frac{1}{2 m^{2}}\left(4 \alpha+H^{\prime \prime}(0)-4\right) c_{1}^{2}=0,
$$

which further provides

$$
H^{\prime \prime}(0)=4(1-\alpha)
$$


Finally, the following asymptotic error constant term is obtained if we insert (42), (44) and (46) in (41)

$$
e_{\lambda+1}=-\frac{c_{1}}{6 m^{3}}\left[\left(24 \alpha^{2}-12 \alpha+\theta-(3 m+15)\right) c_{1}^{2}+6 m c_{2}\right] e_{\lambda}^{4}+O\left(e_{\lambda}^{5}\right),
$$

where $\theta=H^{\prime \prime \prime}(0) \in \mathbb{R}$. The expression (47) demonstrates maximum fourth-order convergence for all $\alpha, \theta, \gamma \in \mathbb{R}$ with three different evaluations of function. Hence, our Scheme (4) has an optimal convergence order as stated in the conjecture given by Kung-Traub.

\section{Some Special Cases of Proposed Scheme}

Here, some of the special cases are generated from proposed scheme, (4) by using different weight functions $H(\tau)$ that satisfies the conditions of Theorems 1-3.

1. Consider $H(\tau)=1+\frac{\tau}{2}+2(1-\alpha) \tau^{2}$, then we have following new method denoted as $P M_{1}$

$$
\begin{aligned}
y_{\lambda} & =x_{\lambda}-m \frac{g\left(x_{\lambda}\right)}{g\left[\mu_{\lambda}, x_{\lambda}\right]} \\
x_{\lambda+1} & =x_{\lambda}+m \frac{g\left(x_{\lambda}\right)}{g\left[\mu_{\lambda}, x_{\lambda}\right]}\left(1+\frac{\zeta}{1-2 \alpha \zeta}\right)\left[\frac{1}{2} \zeta-\left(1+\frac{\tau}{2}+2(1-\alpha) \tau^{2}\right)\right], \alpha \in \mathbb{R} .
\end{aligned}
$$

2. Consider $H(\tau)=\frac{1+\frac{\tau}{2}}{1+\frac{1}{2}(-4+4 \alpha) \tau^{2}}$, then the new method denoted as $P M_{2}$ is obtained as follows:

$$
\begin{aligned}
y_{\lambda} & =x_{\lambda}-m \frac{g\left(x_{\lambda}\right)}{g\left[\mu_{\lambda}, x_{\lambda}\right]} \\
x_{\lambda+1} & =x_{\lambda}+m \frac{g\left(x_{\lambda}\right)}{g\left[\mu_{\lambda}, x_{\lambda}\right]}\left(1+\frac{\zeta}{1-2 \alpha \zeta}\right)\left[\frac{1}{2} \zeta-\left(\frac{1+\frac{\tau}{2}}{1+\frac{1}{2}(-4+4 \alpha) \tau^{2}}\right)\right], \quad \alpha \in \mathbb{R} .
\end{aligned}
$$

3. Consider $H(\tau)=\frac{1+\frac{3}{2} \tau+\frac{1}{2}(5-4 \alpha) \tau^{2}+\tau^{3}}{1+\tau}$, then we have following new method denoted as $P M_{3}$

$$
\begin{aligned}
y_{\lambda} & =x_{\lambda}-m \frac{g\left(x_{\lambda}\right)}{g\left[\mu_{\lambda}, x_{\lambda}\right]}, \\
x_{\lambda+1} & =x_{\lambda}+m \frac{g\left(x_{\lambda}\right)}{g\left[\mu_{\lambda}, x_{\lambda}\right]}\left(1+\frac{\zeta}{1-2 \alpha \zeta}\right)\left[\frac{1}{2} \zeta-\left(\frac{1+\frac{3}{2} \tau+\frac{1}{2}(5-4 \alpha) \tau^{2}+\tau^{3}}{1+\tau}\right)\right], \alpha \in \mathbb{R} .
\end{aligned}
$$

Similarly, many more methods can be created by adopting the following $H(\tau)$ weight functions.
4. $H(\tau)=\left(\frac{1}{2}+\frac{e^{\tau}}{2}+\frac{(7-8 \alpha) \tau^{2}}{4}\right)$.
5. $H(\tau)=\cos (\tau)+\frac{\tau^{2}}{2}+2(1-\alpha) \tau^{2}+\frac{\tau}{2}$.

\section{Numerical Illustration}

In this section, derivative-free proposed Schemes (48)-(50) are verified on some numerical problems. Here, we have made two types of comparisons. Firstly, the results are compared with other existing derivative methods developed by Soleymani et al. [21], and Fiza et al. [22], respectively. Secondly, we compare them with derivative free techniques presented by Hueso et al. [14], Sharma et al. [16], and Sharma et al. [17] respectively. In Hueso et al. technique, we have used the parameter $q=1$ if $m \geq 4$, otherwise $q=2$. All 
the above mentioned existing schemes are listed below:

Soleymani et al. Scheme(SLM):

$$
\begin{aligned}
y_{\lambda} & =x_{\lambda}-\frac{2 m}{m+2} \frac{g\left(x_{\lambda}\right)}{g^{\prime}\left(x_{\lambda}\right)} \\
x_{\lambda+1} & =x_{\lambda}-\frac{g\left(x_{\lambda}\right) g^{\prime}\left(y_{\lambda}\right)}{s_{1}\left(g^{\prime}\left(y_{\lambda}\right)\right)^{2}+s_{2} g^{\prime}\left(x_{\lambda}\right) g^{\prime}\left(y_{\lambda}\right)+s_{3}\left(g^{\prime}\left(x_{\lambda}\right)\right)^{2}},
\end{aligned}
$$

where $s_{1}=\frac{1}{16} m^{3-m}(m+2)^{m}, s_{2}=\frac{8-m(m+2)\left(m^{2}-2\right)}{8 m}, s_{3}=\frac{1}{16}(m-2) m^{m-1}(m+2)^{3-m}$. Fiza et al. Scheme $\left(F M_{1}\right)$ :

$$
\begin{gathered}
y_{\lambda}=x_{\lambda}-m \frac{g\left(x_{\lambda}\right)}{g^{\prime}\left(x_{\lambda}\right)} \\
x_{\lambda+1}=y_{\lambda}-m \frac{u_{\lambda}\left(4 u_{\lambda}+1\right)}{\left(u_{\lambda}+1\right)^{2}} \frac{g\left(x_{\lambda}\right)}{g^{\prime}\left(x_{\lambda}\right)} .
\end{gathered}
$$

Fiza et al. Scheme $\left(F M_{2}\right)$ :

$$
\begin{aligned}
y_{\lambda} & =x_{\lambda}-m \frac{2 g\left(x_{\lambda}\right)}{2 g^{\prime}\left(x_{\lambda}\right)+m g\left(x_{\lambda}\right)}, \\
x_{\lambda+1} & =x_{\lambda}-m u_{\lambda}\left(1+2 u_{\lambda}+\frac{11}{2} u_{\lambda}^{2}\right) \frac{g\left(x_{\lambda}\right)}{g^{\prime}\left(x_{\lambda}\right)+m g\left(x_{\lambda}\right)},
\end{aligned}
$$

where

$$
u_{\lambda}=\left(\frac{g\left(y_{\lambda}\right)}{g\left(x_{\lambda}\right)}\right)^{\frac{1}{m}}
$$

Hueso et al. Scheme $(H M D)$ :

$$
\begin{aligned}
y_{\lambda} & =x_{\lambda}-\frac{2 m}{m+2} \frac{g\left(x_{\lambda}\right)}{g\left[x_{\lambda}, \mu_{\lambda}\right]}, \\
x_{\lambda+1} & =x_{\lambda}-\left(s_{1}+s_{2} H\left(x_{\lambda}, y_{\lambda}\right)+s_{3} H\left(y_{\lambda}, x_{\lambda}\right)+s_{4}\left(H\left(x_{\lambda}, y_{\lambda}\right)^{2}\right) \frac{g\left(x_{\lambda}\right)}{g\left[x_{\lambda}, \mu_{\lambda}\right]},\right.
\end{aligned}
$$

where

$$
\begin{aligned}
\mu_{\lambda} & =x_{\lambda}+\left(g\left(x_{\lambda}\right)\right)^{q}, \quad q \in \mathbb{R}, \\
H\left(x_{\lambda}, y_{\lambda}\right) & =\frac{g\left[x_{\lambda}, y_{\lambda}\right]}{g\left[x_{\lambda}, \mu_{\lambda}\right]} \\
s_{1} & =-\frac{1}{4} m\left(m^{3}+3 m^{2}+2 m-4\right) \\
s_{2} & =\frac{1}{8} m\left(\frac{m}{m+2}\right)^{m}(m+2)^{3} \\
s_{3} & =\frac{1}{8} m^{4}\left(\frac{m}{m+2}\right)^{-m} \\
s_{4} & =0 .
\end{aligned}
$$

Sharma et al. Scheme $\left(S M_{1}\right)$ :

$$
\begin{aligned}
y_{\lambda} & =x_{\lambda}-m \frac{g\left(x_{\lambda}\right)}{g\left[x_{\lambda}, \mu_{\lambda}\right]}, \\
x_{\lambda+1} & =y_{\lambda}-\left(\frac{m}{2} h_{\lambda}\left(1+3 h_{\lambda}\right)\right)\left(1+\frac{1}{v_{\lambda}}\right) \frac{g\left(x_{\lambda}\right)}{g\left[x_{\lambda}, \mu_{\lambda}\right]},
\end{aligned}
$$


where

$$
\begin{aligned}
\mu_{\lambda} & =x_{\lambda}+\gamma g\left(x_{\lambda}\right), \quad \gamma \in \mathbb{R}, \\
u_{\lambda} & =\left(\frac{g\left(y_{\lambda}\right)}{g\left(x_{\lambda}\right)}\right) \frac{1}{m}, \\
v_{\lambda} & =\left(\frac{g\left(\mu_{\lambda}\right)}{g\left(x_{\lambda}\right)}\right)^{\frac{1}{m}}, \\
h_{\lambda} & =\frac{u_{\lambda}}{1+u_{\lambda}} .
\end{aligned}
$$

Sharma et al. Scheme $\left(S M_{2}\right)$ :

$$
\begin{gathered}
y_{\lambda}=x_{\lambda}-m \frac{g\left(x_{\lambda}\right)}{g\left[x_{\lambda}, \mu_{\lambda}\right]}, \\
x_{\lambda+1}=x_{\lambda}-h_{\lambda} \frac{g\left(x_{\lambda}\right)}{g\left[x_{\lambda}, \mu_{\lambda}\right]},
\end{gathered}
$$

where

$$
\begin{aligned}
\mu_{\lambda} & =x_{\lambda}+\gamma g\left(x_{\lambda}\right), \quad \gamma \in \mathbb{R}, \\
r_{\lambda} & =\left(\frac{g\left(y_{\lambda}\right)}{g\left(x_{\lambda}\right)}\right)^{\frac{1}{m}}, \\
s_{\lambda} & =\left(\frac{g\left(y_{\lambda}\right)}{g\left(\mu_{\lambda}\right)}\right)^{\frac{1}{m}}, \\
h_{\lambda} & =r_{\lambda}+m\left(r_{\lambda}\right)^{2}+(m-1) s_{\lambda}+m r_{\lambda} s_{\lambda} .
\end{aligned}
$$

In all the numerical problems, the initial parameter $\gamma=-0.01$, is considered. The numerical problems have been performed on software Mathematica 10 using 5000 multiple precision digits of mantissa with stopping criterion $\left|x_{\lambda}-x_{\lambda-1}\right|+\left|g\left(x_{\lambda}\right)\right| \leq 100$. To check the better performance of proposed method, we have displayed the errors between two consecutive iterations $e_{\lambda}=\left|x_{\lambda}-x_{\lambda-1}\right|$, absolute residual error $\left|g\left(x_{\lambda}\right)\right|$ at $(\lambda)$ th iteration, approximate computational order of convergence (ACOC) denoted as $\rho$, and the number of iterations of each method that satisfies the stopping criterion in the Tables 1-4. The following formula is used to evaluate the approximate computational order of convergence $(\mathrm{ACOC})$

$$
\rho=\frac{\ln \frac{\left|x_{\lambda+1}-x_{\lambda}\right|}{\left|x_{\lambda}-x_{\lambda-1}\right|}}{\ln \frac{\left|x_{\lambda}-x_{\lambda-1}\right|}{\left|x_{\lambda-1}-x_{\lambda-2}\right|}}, \quad \text { for each } \lambda=2,3, \ldots
$$

Further, the iterative procedure is stopped after three iterations and each problem is tested on different initial values. Notice that the meaning of $b( \pm a)$ is $b \times 10^{ \pm a}$ in all the tables.

Example 1. Consider the Van der waal equation of ideal gas [23]

$$
\left(P+\frac{a n^{2}}{V^{2}}\right)(V-n b)=n R T
$$

which explains the nature of real gas by taking parameters $a, b$ of a particular gas. Other parameters $n, R$, and $T$ are obtained with parameters $a$, and $b$. So, we have the following nonlinear equations in terms of volume of gas $(V)$ which is represented as $x$ by

$$
g_{1}(x)=x^{3}-5.22 x^{2}+9.0825 x-5.2675 .
$$

The desired root is $\eta=1.75$ of multiplicity $m=3$. Table 1 depicts the performance of different iterative schemes with initial guess $x_{0}=1.9$. The number of iterations utilized by different methods are shown in Table 5. The proposed methods $P M_{1}, P M_{2}$ and $P M_{3}$ converges to root much 
faster than the derivative methods $S L M, F M_{1}$ and $F M_{2}$ and derivative-free methods $H M D, S M_{1}$ and $S M_{2}$.

Table 1. Numerical results of Example 1.

\begin{tabular}{cccccc}
\hline Schemes & $\left|e_{2}\right|$ & $\left|e_{3}\right|$ & $\left|e_{4}\right|$ & $\left|g\left(e_{4}\right)\right|$ & $\rho$ \\
\hline$S L M$ & $1.4(-2)$ & $1.4(-4)$ & $5.5(-12)$ & $4.6(-84)$ & 4.000 \\
\hline$F M 1$ & $1.9(-2)$ & $5.7(-4)$ & $4.6(-9)$ & $1.4(-59)$ & 4.000 \\
\hline$F M 2$ & $1.4(-2)$ & $7.1(-5)$ & $1.2(-14)$ & $7.3(-55)$ & 4.000 \\
\hline$H M D$ & $1.5(-2)$ & $1.8(-4)$ & $1.8(-11)$ & $1.1(-79)$ & 4.000 \\
\hline$S M_{1}$ & $1.9(-2)$ & $5.7(-4)$ & $4.6(-9)$ & $1.3(-59)$ & 4.000 \\
\hline$S M_{2}$ & $1.6(-2)$ & $2.81(-4)$ & $1.5(-10)$ & $5.0(-72)$ & 4.000 \\
\hline$P M_{1}(\alpha=0)$ & $1.5(-2)$ & $1.8(-4)$ & $1.5(-11)$ & $1.9(-80)$ & 4.000 \\
$\alpha=\frac{1}{2}$ & $1.5(-2)$ & $1.6(-4)$ & $1.0(-11)$ & $8.8(-82)$ & 4.000 \\
$\alpha=1$ & $1.2(-2)$ & $4.9(-5)$ & $4.1(-14)$ & $1.2(-101)$ & 4.000 \\
\hline$P M_{2}(\alpha=0)$ & $1.3(-2)$ & $9.9(-5)$ & $1.1(-12)$ & $7.5(-90)$ & 4.000 \\
$\alpha=\frac{1}{2}$ & $1.4(-2)$ & $1.3(-4)$ & $3.5(-12)$ & $1.4(-85)$ & 4.000 \\
$\alpha=1$ & $1.2(-2)$ & $4.9(-5)$ & $4.1(-14)$ & $1.2(-101)$ & 4.000 \\
\hline$P M_{3}(\alpha=0)$ & $1.6(-2)$ & $2.2(-4)$ & $5.1(-11)$ & $5.9(-76)$ & 4.000 \\
$\alpha=\frac{1}{2}$ & $1.5(-2)$ & $1.6(-4)$ & $1.0(-11)$ & $8.8(-82)$ & 4.000 \\
$\alpha=1$ & $1.1(-2)$ & $2.9(-5)$ & $1.6(-15)$ & $5.4(-114)$ & 4.000 \\
\hline
\end{tabular}

Example 2. Consider the planck's radiation equation that determines the spectral density of electromagnetic radiations released by a black-body at a given temperature, and at thermal equilibrium [24] as

$$
G(y)=\frac{8 \pi c h y^{-5}}{e^{\frac{c h}{k T}}-1},
$$

where $T, y, k, h$, and $c$ denotes the absolute temperature of the black-body, wavelength of radiation, Boltzmann constant, Plank's constant, and speed of light in the medium (vacuum), respectively. To evaluate the wavelength $y$ which results to the maximum energy density $G(y)$, set $G^{\prime}(y)=0$. We obtained the following equation:

$$
\frac{\left(\frac{c h}{y k T}\right) e^{\frac{c h}{y k T}}}{e^{\frac{c h}{y k T}}-1}=5 .
$$

Further, the nonlinear equation is formulated by setting $x=\frac{c h}{y k T}$ as follows:

$$
g_{2}(x)=\left(e^{-x}-1+\frac{x}{5}\right)^{m} .
$$

The exact root is $\eta=4.96511423174427630369$ of multiplicity $m=4$ and with this root one can easily find the wavelength $y$ form the relation $x=\frac{c h}{y k T}$. Planck's problem was tested with initial guess $x_{0}=5.5$ and results are demonstrated in Table 2. Table 5 represents the comparison analysis of iterations vs schemes for this Example. 
Table 2. Numerical results of Example 2.

\begin{tabular}{cccccc}
\hline Schemes & $\left|e_{2}\right|$ & $\left|e_{3}\right|$ & $\left|e_{4}\right|$ & $\left|g\left(e_{4}\right)\right|$ & $\rho$ \\
\hline$S L M$ & $4.9(-5)$ & $5.7(-21)$ & $1.1(-84)$ & $2.1(-1359)$ & 4.000 \\
\hline$F M 1$ & $6.3(-6)$ & $2.7(-25)$ & $9.3(-103)$ & $3.4(-1651)$ & 4.000 \\
\hline$F M 2$ & $9.6(-3)$ & $9.5(-5)$ & $3.9(-17)$ & $8.1(-135)$ & 6.022 \\
\hline$H M D$ & $1.1(-3)$ & $1.7(-14)$ & $1.1(-57)$ & $2.3(-922)$ & 4.000 \\
\hline$S M_{1}$ & $6.4(-6)$ & $2.7(-25)$ & $9.4(-103)$ & $3.9(-1651)$ & 4.000 \\
\hline$S M_{2}$ & $5.6(-6)$ & $1.3(-25)$ & $4.4(-104)$ & $9.3(-1673)$ & 4.000 \\
\hline$P M_{1}(\alpha=0)$ & $5.3(-6)$ & $9.7(-26)$ & $1.1(-104)$ & $2.0(-1682)$ & 4.000 \\
$\alpha=\frac{1}{2}$ & $5.3(-6)$ & $9.7(-26)$ & $1.1(-104)$ & $1.8(-1682)$ & 4.000 \\
$\alpha=1$ & $4.9(-6)$ & $6.8(-26)$ & $2.5(-105)$ & $4.7(-1693)$ & 4.000 \\
\hline$P M_{2}(\alpha=0)$ & $5.1(-6)$ & $8.1(-26)$ & $5.3(-105)$ & $1.1(-1687)$ & 4.000 \\
$\alpha=\frac{1}{2}$ & $5.2(-6)$ & $8.9(-26)$ & $7.7(-105)$ & $4.8(-1685)$ & 4.000 \\
$\alpha=1$ & $4.9(-6)$ & $6.8(-26)$ & $2.5(-105)$ & $4.7(-1693)$ & 4.000 \\
\hline$P M_{3}(\alpha=0)$ & $5.4(-6)$ & $1.1(-25)$ & $2.2(-104)$ & $1.7(-1677)$ & 4.000 \\
$\alpha=\frac{1}{2}$ & $5.3(-6)$ & $9.7(-26)$ & $1.1(-104)$ & $1.8(-1682)$ & 4.000 \\
$\alpha=1$ & $4.8(-6)$ & $5.7(-26)$ & $1.1(-105)$ & $1.4(-1698)$ & 4.000 \\
\hline
\end{tabular}

Example 3. Finding the eigenvalues of a large matrix whose order is greater than 4, we need to solve its characteristic equation. The determination of roots of such higher-order characteristic equations is a difficult task if we apply the linear algebra approach. So, one of the best ways is to use numerical techniques. Now, consider the following square matrix of order 9:

$$
A=\frac{1}{8}\left(\begin{array}{ccccccccc}
-12 & 0 & 0 & 19 & -19 & 76 & -19 & 18 & 437 \\
-64 & 24 & 0 & -24 & 24 & 64 & -8 & 32 & 376 \\
-16 & 0 & 24 & 4 & -4 & 16 & -4 & 8 & 92 \\
-40 & 0 & 0 & -10 & 50 & 40 & 2 & 20 & 242 \\
-4 & 0 & 0 & -1 & 41 & 4 & 1 & 2 & 25 \\
-40 & 0 & 0 & 18 & -18 & 104 & -18 & 20 & 462 \\
-84 & 0 & 0 & -29 & 29 & 84 & 21 & 42 & 501 \\
16 & 0 & 0 & -4 & 4 & -16 & 4 & 16 & -92 \\
0 & 0 & 0 & 0 & 0 & 0 & 0 & 0 & 24
\end{array}\right)
$$

whose characteristic equation is modeled into nonlinear equation as 6

$$
g_{3}(x)=x^{9}-29 x^{8}+349 x^{7}-2261 x^{6}+8455 x^{5}-17663 x^{4}+15927 x^{3}+6993 x^{2}-24732^{x}+12960 .
$$

The root of this equation is $\eta=3$ with multiplicity $m=4$. Table 3 depicts the better performance of proposed schemes in comparison of existing techniques by taking an initial guess $x_{0}=2.8$. The total number of iterations required to converge the roots are shown in Table 5. 
Table 3. Numerical results of Example 3.

\begin{tabular}{cccccc}
\hline Schemes & $\left|e_{2}\right|$ & $\left|e_{3}\right|$ & $\left|e_{4}\right|$ & $\left|g\left(e_{4}\right)\right|$ & $\rho$ \\
\hline$S L M$ & $1.3(-5)$ & $4.4(-17)$ & $6.5(-67)$ & $6.4(-1061)$ & 4.000 \\
\hline$F M 1$ & $9.9(-5)$ & $1.7(-17)$ & $1.4(-68)$ & $9.4(-1088)$ & 4.000 \\
\hline$F M 2$ & $3.4(-2)$ & $6.9(-8)$ & $2.5(-15)$ & $3.4(-237)$ & 1.330 \\
\hline$H M D$ & $5.1(-2)$ & $8.7(-3)$ & $3.9(-6)$ & $6.5(-74)$ & 4.000 \\
\hline$S M_{1}$ & $7.7(-5)$ & $6.2(-18)$ & $2.6(-70)$ & $4.2(-1115)$ & 4.000 \\
\hline$S M_{2}$ & $6.2(-5)$ & $1.6(-18)$ & $7.8(-73)$ & $2.1(-1156)$ & 4.000 \\
\hline$P M_{1}(\alpha=0)$ & $6.5(-5)$ & $1.4(-18)$ & $3.4(-73)$ & $1.3(-1162)$ & 4.000 \\
$\alpha=\frac{1}{2}$ & $6.6(-5)$ & $1.5(-18)$ & $4.6(-73)$ & $1.2(-1160)$ & 4.000 \\
$\alpha=1$ & $6.4(-5)$ & $9.1(-19)$ & $3.8(-74)$ & $1.2(-1178)$ & 4.000 \\
\hline$P M_{2}(\alpha=0)$ & $6.3(-5)$ & $1.1(-18)$ & $9.1(-74)$ & $3.9(-1172)$ & 4.000 \\
$\alpha=\frac{1}{2}$ & $6.5(-5)$ & $1.3(-18)$ & $2.4(-73)$ & $3.5(-1165)$ & 4.000 \\
$\alpha=1$ & $6.4(-5)$ & $9.1(-19)$ & $3.8(-74)$ & $1.2(-1178)$ & 4.000 \\
\hline$P M_{3}(\alpha=0)$ & $6.6(-5)$ & $1.8(-18)$ & $1.1(-72)$ & $1.9(-1154)$ & 4.000 \\
$\alpha=\frac{1}{2}$ & $6.6(-5)$ & $1.5(-18)$ & $4.6(-73)$ & $1.2(-1160)$ & 4.000 \\
$\alpha=1$ & $6.3(-5)$ & $6.2(-19)$ & $6.2(-75)$ & $1.1(-1191)$ & 4.000 \\
\hline
\end{tabular}

Example 4. Consider another standard academic problem as follows:

$g_{4}(x)=\left(\tan ^{-1}\left(\frac{\sqrt{5}}{2}\right)-\tan ^{-1}\left(\sqrt{x^{2}-1}\right)+\sqrt{6}\left(\tan ^{-1}\left(\sqrt{\frac{x^{2}-1}{6}}\right)-\tan ^{-1}\left(\frac{1}{2} \sqrt{\frac{5}{6}}\right)\right)-\frac{11}{63}\right)^{4}$

which has root $\eta=1.8411027704926161$ of multiplicity 4 . The results are obtained on initial guess $x_{0}=1.5$ and shown in Table 4. Table 5 shows the iteration comparison for this standard nonlinear equation.

Table 4. Numerical results of Example 4.

\begin{tabular}{cccccc}
\hline Schemes & $\left|e_{2}\right|$ & $\left|e_{3}\right|$ & $\left|e_{4}\right|$ & $\left|g\left(e_{4}\right)\right|$ & $\rho$ \\
\hline$S L M$ & $1.1(-3)$ & $1.1(-14)$ & $1.2(-58)$ & $3.2(-917)$ & 4.000 \\
\hline$F M 1$ & $2.7(-5)$ & $1.0(-20)$ & $2.3(-82)$ & $8.0(-1315)$ & 4.000 \\
\hline$F M 2$ & $2.3(-1)$ & $3.7(-4)$ & $1.1(-7)$ & $5.5(-116)$ & 1.328 \\
\hline$H M D$ & $9.3(-3)$ & $4.1(-9)$ & $1.6(-34)$ & $1.4(-543)$ & 4.000 \\
\hline$S M_{1}$ & $2.7(-5)$ & $1.1(-20)$ & $2.7(-82)$ & $6.3(-1314)$ & 4.000 \\
\hline$S M_{2}$ & $2.7(-5)$ & $7.4(-21)$ & $4.4(-83)$ & $4.7(-1327)$ & 4.000 \\
\hline$P M_{1}(\alpha=0)$ & $2.7(-5)$ & $6.1(-21)$ & $1.7(-83)$ & $6.4(-1334)$ & 4.000 \\
$\alpha=\frac{1}{2}$ & $2.7(-5)$ & $6.1(-21)$ & $1.7(-83)$ & $6.4(-1334)$ & 4.000 \\
$\alpha=1$ & $2.7(-5)$ & $4.9(-21)$ & $5.5(-84)$ & $3.2(-1342)$ & 4.000 \\
\hline$P M_{2}(\alpha=0)$ & $2.7(-5)$ & $5.5(-21)$ & $9.9(-84)$ & $7.6(-1338)$ & 4.000 \\
$\alpha=\frac{1}{2}$ & $2.7(-5)$ & $5.8(-21)$ & $1.3(-83)$ & $7.8(-1336)$ & 4.000 \\
$\alpha=1$ & $2.7(-5)$ & $4.9(-21)$ & $5.5(-84)$ & $3.2(-1342)$ & 4.000 \\
\hline$P M_{3}(\alpha=0)$ & $2.7(-5)$ & $6.8(-21)$ & $2.8(-83)$ & $2.3(-1330)$ & 4.000 \\
$\alpha=\frac{1}{2}$ & $2.7(-5)$ & $6.1(-21)$ & $1.7(-83)$ & $6.4(-1334)$ & 4.000 \\
$\alpha=1$ & $2.7(-5)$ & $4.3(-21)$ & $2.8(-84)$ & $3.7(-1347)$ & 4.000 \\
\hline
\end{tabular}


Table 5. Iterations comparison of different schemes.

\begin{tabular}{ccccc}
\hline Schemes & Example 1 & Example 2 & Example 3 & Example 4 \\
\hline$S L M$ & 6 & 5 & 5 & 5 \\
\hline$F M_{1}$ & 6 & 4 & 5 & 5 \\
\hline$F M_{2}$ & 6 & 6 & 6 & 7 \\
\hline$H M D$ & 6 & 5 & 7 & 5 \\
\hline$S M_{1}$ & 6 & 4 & 5 & 5 \\
\hline$S M_{2}$ & 6 & 4 & 5 & 5 \\
\hline$M_{1}(\alpha=0)$ & 6 & 4 & 5 & 5 \\
$\alpha=\frac{1}{2}$ & 6 & 4 & 5 & 5 \\
$\alpha=1$ & 6 & 4 & 5 & 5 \\
\hline$M_{1}(\alpha=0)$ & 6 & 4 & 5 & 5 \\
$\alpha=\frac{1}{2}$ & 6 & 4 & 5 & 6 \\
$\alpha=1$ & 6 & 4 & 5 & 5 \\
\hline$M_{1}(\alpha=0)$ & 6 & 4 & 5 & 5 \\
$\alpha=\frac{1}{2}$ & 6 & 4 & 5 & 5 \\
$\alpha=1$ & 6 & 4 & 5 & \\
\hline
\end{tabular}

It seems that the number of iterations is equal as compared with earlier methods in Table 5 for each example. Tables 1-4 demonstrates the less residual functional error of the constructed methods in the comparison of existing schemes with the same number of iterations. Moreover, the order of convergence in each numerical except Example 1 is not attained by $F M_{2}$ method, so it is denoted as -.

\section{Dynamical Planes of (48) and (49)}

In this section, we will show some dynamical planes, as it appears in different studies such as [25-30], associated with the families (48) and (49), but applied to the polynomial

$$
p(z)=(z-1)^{2}(z+1)
$$

with a double root $z=1$ and a simple one $z=-1$ for different values of $\alpha$ and $\gamma$.

We paint in red and blue the convergence after 100 iteration to the roots of the polynomial with a tolerance of $10^{-3}$ in another case the point is painted in black.

We see in Figures 1 and 2 the dynamical behavior of Method (48) for different values of $\alpha$ and $\gamma$.

As a consequence, it is clear that depending on the values of $\alpha$ and $\gamma$ the convergence to the simple root can be lost but the convergence to the double one is not lost with family (48).

We see in Figures 3 and 4 the dynamical behavior of Method (48) for different values of $\alpha$ and $\gamma$. 


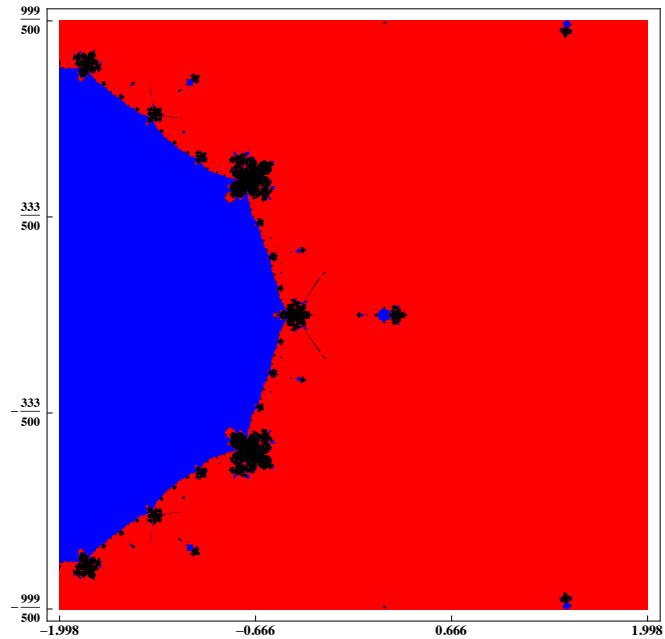

(a) Method (48) with $\alpha=1, \gamma=0.1$

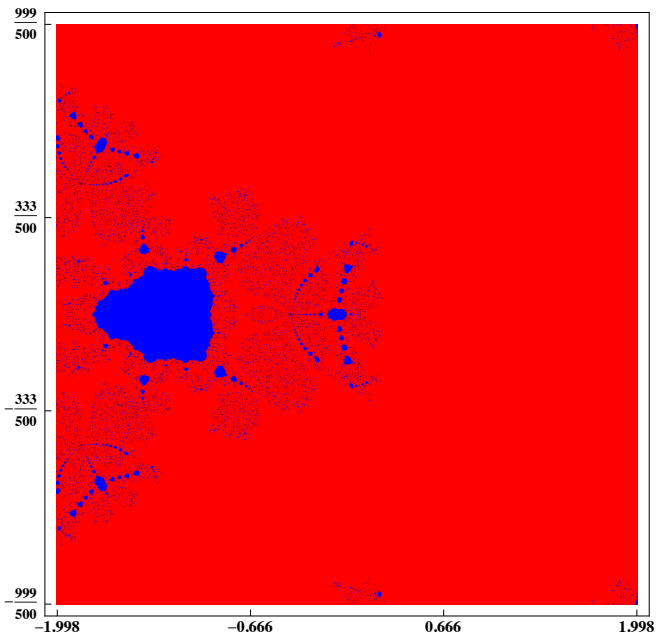

(b) Method (48) with $\alpha=0.1, \gamma=0.5$

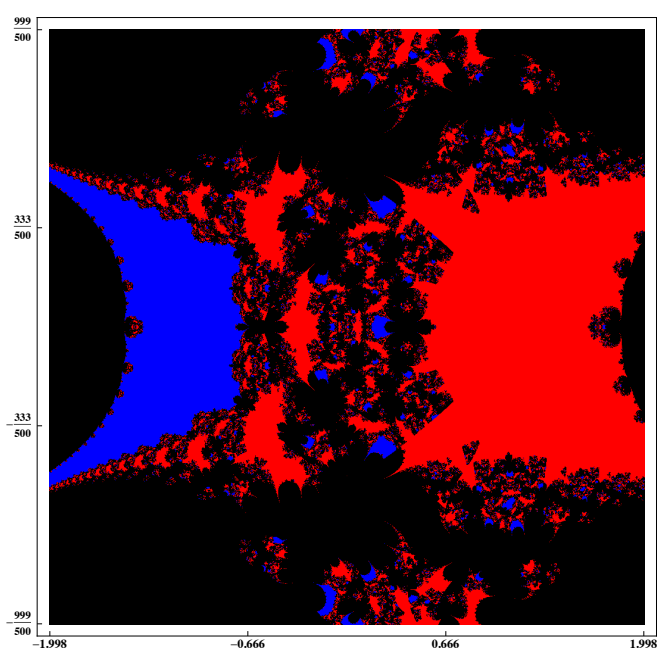

(c) Method (48) with $\alpha=1, \gamma=0.5$

Figure 1. Basins of attraction associated to the method (48) applied to polynomial $p(z)=(z-1)^{2}(z+$ 1) with different values for tolerance.

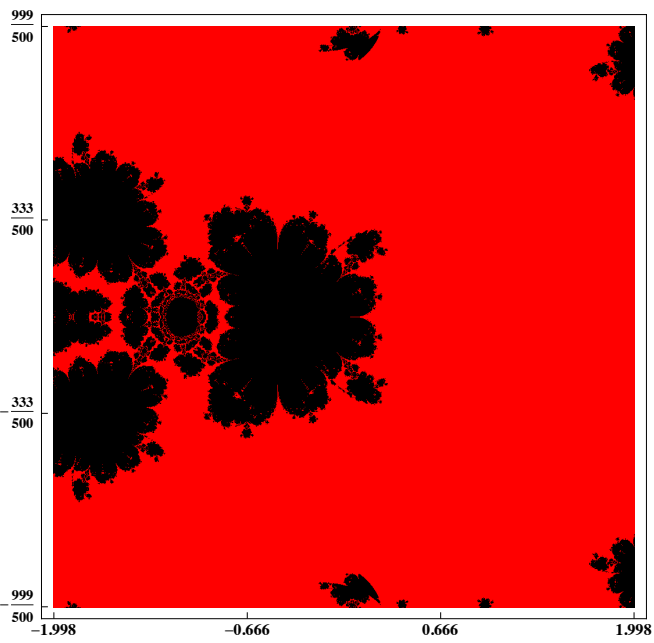

(a) Method (48) with $\alpha=0.1, \gamma=0.1$

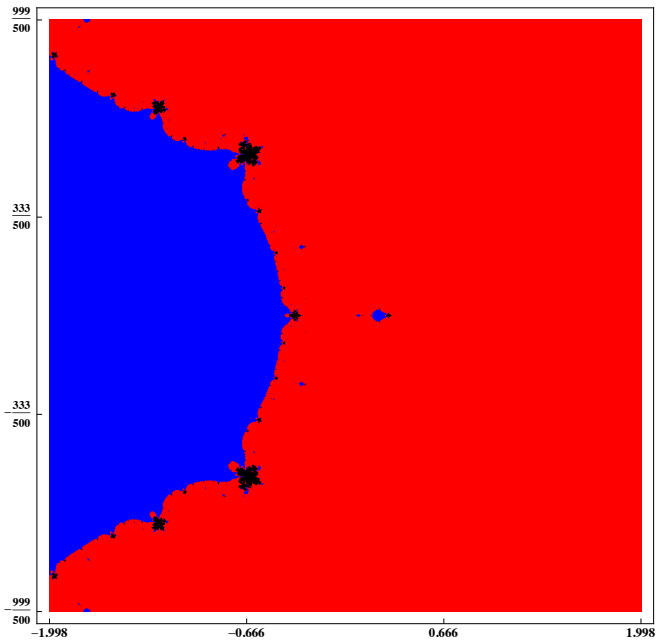

(b) Method (48) with $\alpha=1, \gamma=0.01$

Figure 2. Cont. 


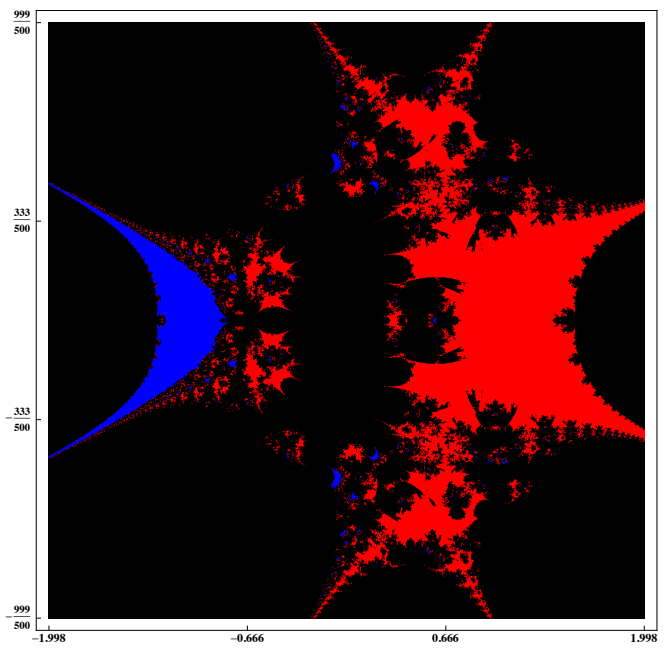

(c) Method (48) with $\alpha=1, \gamma=1$

Figure 2. Basins of attraction associated to the method (48) applied to polynomial $p(z)=(z-1)^{2}(z+$ 1) with different values for tolerance.

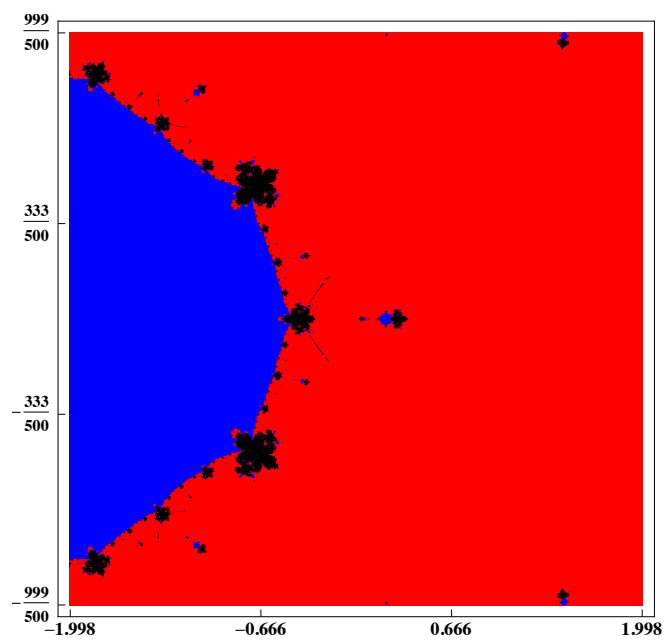

(a) Method (49) with $\alpha=1, \gamma=0.1$

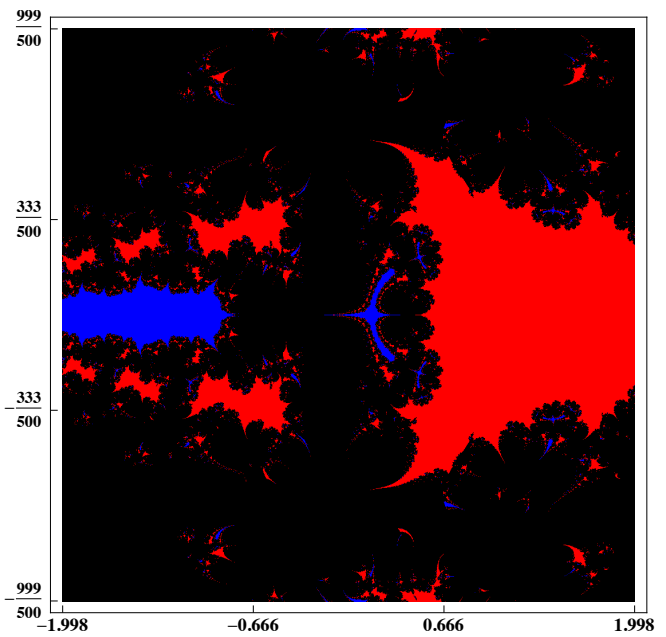

(b) Method (49) with $\alpha=0.1, \gamma=0.5$

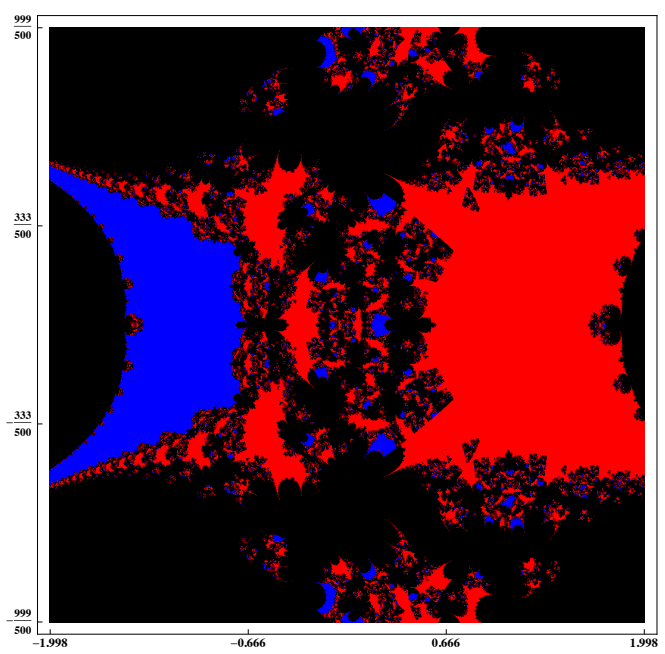

(c) Method (49) with $\alpha=1, \gamma=0.5$

Figure 3. Basins of attraction associated to the method (49) applied to polynomial $p(z)=(z-1)^{2}(z+$ 1) with different values for tolerance. 


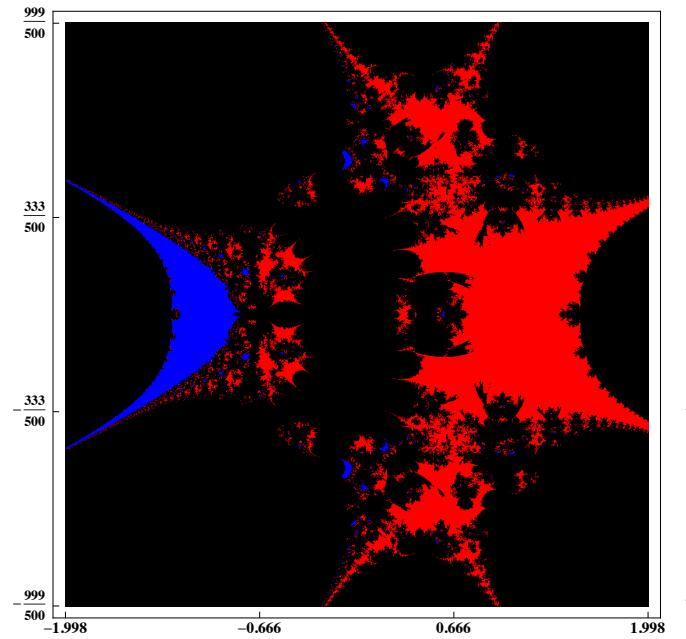

(a) Method (49) with $\alpha=1, \gamma=1$

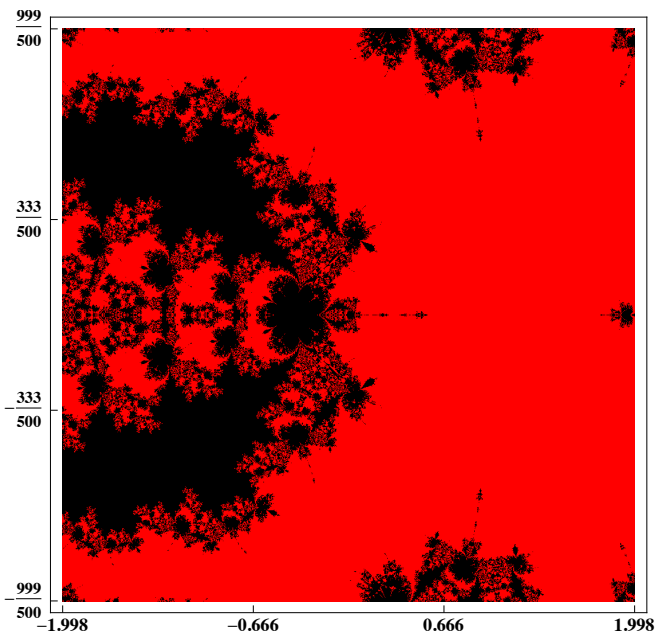

(b) Method (49) with $\alpha=2, \gamma=0.1$

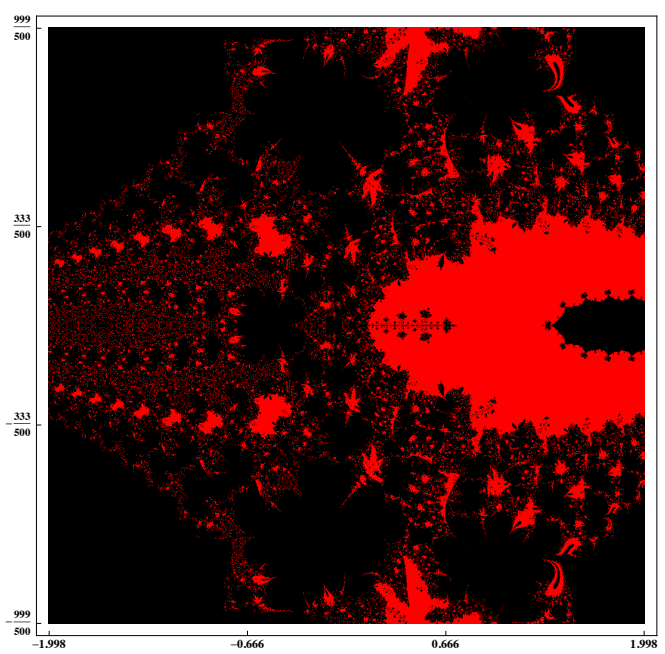

(c) Method (49) with $\alpha=2, \gamma=0.5$

Figure 4. Basins of attraction associated to the method (49) applied to polynomial $p(z)=(z-1)^{2}(z+$ 1) with different values for tolerance.

Consequently, from Figures 3 and 4, it is clear that depending on the values of $\alpha$ and $\gamma$ the convergence to the simple root can be lost but the convergence to the double one is not lost with family (49) and also for $\alpha=0.1$ and $\gamma=0.5$ each point of the plane converge to one of the roots.

\section{Conclusions}

The higher-order optimal derivative -free family of Chebyshev-Halley's iterative method have been introduced to solve the nonlinear equations of multiple roots. The fourth-order convergence method has been constructed with one parameter $\alpha$ and one weight function. The convergence of proposed techniques is analyzed. To verify the theoretical efficiency of the suggested method some numerical illustrations are performed and obtained results represent the better results vs earlier methods. Finally, dynamical planes of proposed methods are discussed which shows better stability.

Author Contributions: Conceptualization, R.B., S.B., Á.A.M. and A.M.; methodology, R.B., S.B., Á.A.M. and A.M.; software, R.B., S.B., Á.A.M. and A.M. validation, R.B., S.B., Á.A.M. and A.M.; formal analysis, R.B., S.B., Á.A.M. and A.M. investigation, R.B., S.B., Á.A.M. and A.M.; resources, R.B., S.B., Á.A.M. and A.M.; data curation, R.B., S.B., Á.A.M. and A.M.; writing-original draft preparation, R.B., S.B., Á.A.M. and A.M.; writing-review and editing, R.B., S.B., Á.A.M. and A.M.; visualization, 
R.B., S.B., Á.A.M. and A.M.; supervision, R.B., S.B., Á.A.M. and A.M.; project administration, R.B., S.B., Á.A.M. and A.M.; funding acquisition, R.B., S.B., Á.A.M. and A.M. All authors have read and agreed to the published version of the manuscript.

Funding: This research was funded by Deanship of Scientific Research, King Saud University grant number D-13-130-1442, Fundación Séneca grant number 20928/PI/18 and Ministerio de Ciencia, Innovación y Universidades grant number PGC2018-095896-B-C21.

Institutional Review Board Statement: Not applicable.

Informed Consent Statement: Not applicable.

Data Availability Statement: Not applicable.

Acknowledgments: The research of the author A.A.M. was funded in part by Programa de Apoyo a la investigación de la fundación Séneca-Agencia de Ciencia y Tecnología de la Región de Murcia 20928/PI/18 and by Spanish MCINN PGC2018-095896-B-C21.

Conflicts of Interest: The authors declare no conflict of interest.

\section{References}

1. Li, S.; Liao, X.; Cheng, L. A new fourth-order iterative method for finding multiple roots of nonlinear equations. Appl. Math. Comput. 2009, 215, 1288-1292.

2. Sharma, J.R.; Sharma, R. Modified Jarratt method for computing multiple roots. Appl. Math. Comput. 2010, $217,878-881$. [CrossRef]

3. Zhou, X.; Chen, X.; Song, Y. Constructing higher-order methods for obtaining the multiple roots of nonlinear equations. J. Comput. Appl. Math. 2011, 235, 4199-4206. [CrossRef]

4. Neta, B.; Chun, C.; Scott, M. On the development of iterative methods for multiple roots. Appl. Math. Comput. 2013, 224, 358-361. [CrossRef]

5. Behl, R.; Cordero, A.; Motsa, S.S.; Torregrosa, J.R. On developing fourth-order optimal families of methods for multiple roots and their dynamics. Appl. Math. Comput. 2015, 265, 520-532. [CrossRef]

6. Behl, R.; Cordero, A.; Motsa, S.S.; Torregrosa, J.R.; Kanwar, V. An optimal fourth-order family of methods for multiple roots and its dynamics. Numer. Algorithms 2016, 71, 775-796. [CrossRef]

7. Geum, Y.H.; Kim, Y.I.; Neta, B. Constructing a family of optimal eighth-order modified Newton-type multiple-zero finders along with the dynamics behind their purely imaginary extraneous fixed points. J. Comput. Appl. Math. 2018, 333, 131-156. [CrossRef]

8. Kansal, M.; Behl, R.; Mahnashi, M.A.A.; Mallawi, F. Modified Optimal Class of Newton-Like Fourth-Order Methods for Multiple Roots. Symmetry 2019, 11, 526. [CrossRef]

9. Regmi, S. Optimized Iterative Methods with Applications in Diverse Disciplines; Nova Science Publisher: New York, NY, USA, 2021.

10. Ostrowski, A.M. Solutions of Equations and System of Equations; Academic Press: New York, NY, USA, 1964.

11. Traub, J.F. Iterative Methods for the Solution of Equations; Prentice-Hall Series in Automatic Computation: Englewood Cliffs, NJ, USA, 1964.

12. Petković, M.S.; Neta, B.; Petković, L.D.; Džunić, J. Multipoint Methods for Solving Nonlinear Equations; Academic Press: New York, NY, USA, 2012.

13. Hernádez, M.A.; Salanova, M.A. A family of chebyshev type methods in banach spaces. Int. J. Comput. Methods 1996, 61, 145-154. [CrossRef]

14. Hueso, J.L.; Martínez, E.; Teruel, C. Determination of multiple roots of nonlinear equations and applications. Math. Chem. 2015, 53, 880-892. [CrossRef]

15. Kumar, D.; Sharma, J.R.; Argyros, I.K. Optimal one-point iterative function free from derivatives for multiple roots. Mathematics 2020, 8, 709. [CrossRef]

16. Sharma, J.R.; Kumar, S.; Jäntschi, L. On Derivative Free Multiple-Root Finders with Optimal Fourth Order Convergence. Mathematics 2020, 8, 1091. [CrossRef]

17. Sharma, J.R.; Kumar, S.; Jäntschi, L. On a class of optimal fourth order multiple root solvers without using derivatives. Symmetry 2019, 11, 1452. [CrossRef]

18. Sharma, J.R.; Kumar, S.; Argyros, I.K. Development of optimal eighth order derivative-free methods for multiple roots of nonlinear equations. Symmetry 2019, 11, 766. [CrossRef]

19. Kung, H.T.; Traub, J.F. Optimal order of one-point and multipoint iteration. J. Assoc. Comput. Mach. 1974, 21, 643-651. [CrossRef]

20. Ahlfors, I.V. Complex Analysis; McGraw-Hill Book, Inc.: New York, NY, USA, 1979.

21. Soleymani, F.; Babajee, D.K.R.; Lotfi, T. On a numerical technique for finding multiple zeros and its dynamics. J. Egypt. Math. Soc. 2013, 21, 346-353. [CrossRef]

22. Zafar, F.; Cordero, A.; Torregrosa, J.R. Stability analysis of a family of optimal fourth-order methods for multiple roots. Numer. AlgoriTheorem. 2018, 81, 947-981. [CrossRef] 
23. Behl, R.; Zafar, F.; Alshormani, A.S.; Junjua, M.U.D.; Yasmin, N. An optimal eighth-order scheme for multiple zeros of unvariate functions. Int. J. Comput. Methods 2019, 16, 1-16. [CrossRef]

24. Bradie, B. A Friendly Introduction to Numerical Analysis; Pearson Education Inc.: New Delhi, India, 2006.

25. Lotfi, T.; Magreñán, Á.A.; Mahdiani, K.; Javier Rainer, J. A variant of Steffensen-King's type family with accelerated sixth-order convergence and high efficiency index: Dynamic study and approach. Appl. Math. Comput. 2015, 252, 347-353. [CrossRef]

26. Gutiérrez, J.M.; Magreñán, Á.A.; Varona, J.L. The "gauss-Seidelization" of iterative methods for solving nonlinear equations in the complex plane. Appl. Math. Comput. 2011, 218, 2467-2479. [CrossRef]

27. Amat, S.; Busquier, S.; Bermúdez, C.; Magreñán, Á.A. On the election of the damped parameter of a two-step relaxed Newton-type method. Nonlinear Dyn. 2016, 84, 9-18. [CrossRef]

28. Magreñán, Á.A.; Gutiérrez, J.M. Real dynamics for damped Newton's method applied to cubic polynomials. J. Comput. Appl. Math. 2015, 275, 527-538. [CrossRef]

29. Cordero, A.; Feng, L.; Magreñán, Á.A.; Torregrosa, J.R. A new fourth-order family for solving nonlinear problems and its dynamics. J. Math. Chem. 2015, 53, 893-910. [CrossRef]

30. Magreñán, Á.A. Different anomalies in a Jarratt family of iterative root-finding methods. Appl. Math. Comput. 2014, 233, 29-38. 